\title{
Single-leg choice-based revenue management: a robust optimisation approach
}

\author{
Received (in revised form): 14th February 2016
}

\author{
Dirk Sierag ${ }^{\mathrm{a}, \mathrm{b}}$ and Rob van der Mei ${ }^{\mathrm{a}, \mathrm{b}}$ \\ ${ }^{a}$ Stochastics Department, CWI, Science Park 123, 1098 XG Amsterdam, The Netherlands; and ${ }^{b}$ Faculty of \\ Exact Sciences, VU University Amsterdam, Amsterdam, The Netherlands
}

Dirk Sierag is a mathematician with special interest in operations research and financial mathematics. He holds a PhD position in dynamic pricing and revenue management at the Center for Mathematics and Computer Science (CWI), Amsterdam. His research emphasises on customer choice behaviour, (online) ratings and reviews, overbooking and cancellations, and derivative pricing.

Rob van der Mei is a full professor at the VU University Amsterdam, the head of the research theme Logistics and the Industrial Liaison Officer at CWI. Before going to academia, he has been working for over a decade as a consultant and researcher in the ICT industry, working for PTT, KPN, AT\&T Bell Labs and TNO ICT. He is the initiator and leader of the project 'From Reactive to proactive Planning of Ambulance Services (REPRO), and a co-founder of the spin-off company Stokhos Emergency Mathematics. His research interests include performance modelling and scalability analysis of ICT systems, logistics, grid computing, revenue management, military operations research, sensor networks, call centers, queueing theory and applications of Big Data. He is the co-author of some 150 papers in journals and refereed proceedings.

Correspondence: Dirk Sierag, Faculty of Exact Sciences, VU University Amsterdam, Amsterdam, The Netherlands

E-mail: d.d.sierag@cwi.nl

\begin{abstract}
A popular trend in revenue management captures the behaviour of customers that choose between different available products. The provided solution methods assume that there is no uncertainty in the parameters of the model. However, in practice the parameters may be uncertain, e.g., because of estimation errors. A relatively recent field of optimisation that takes into account uncertainty in the optimisation procedure is robust optimisation. Robust optimisation methods provide solutions where the worstcase scenario is optimised, taking into account uncertainty in parameters. This paper studies a robust optimisation approach to single-leg choice-based revenue management based on Talluri and van Ryzin (Manag Sci 50:15-33, 2004) and Sierag et al (Eur J Oper Res 246:170-185, 2015). The problem is modelled as a Markov decision process and solved using dynamic programming. This paper uses $\phi$-divergence uncertainty sets to model the probability vectors of general choice-models. Novel robust optimisation techniques are applied to the dynamic program, taking into account uncertainty in the parameters. An important yet surprising insight from the numerical results is that the robust solution method performs better for smaller inventory than for larger inventory. Moreover, the robust solution method shows great performance when knowledge on cancellation behaviour is lacking: on average the expected reward then improves by $2.5-3.25$ per cent.

Journal of Revenue and Pricing Management (2016). doi:10.1057/s41272-016-0069-6
\end{abstract}

Keywords: Revenue management; dynamic pricing; customer choice models; Markov decision processes; robust optimisation 


\section{INTRODUCTION}

A popular trend in revenue management models captures the behaviour of customers that choose between different available products. The renowned paper by Talluri and van Ryzin (2004) combined revenue management models with customer choice models. Many contributions to the body of literature on customer choice models have been made, e.g., better solution methods Strauss and Talluri (2012), network models that take into account multiple night stays/multiple flight legs Liu and van Ryzin (2008), and recently Sierag et al (2015) extended the model to include cancellations, to which the model of this paper is closely related. The solution methods used in these models often assume that the parameters of the model are known. However, in practice the true parameter values are unknown and have to be estimated from data. Estimating the parameters of a customer choice model requires more data than only sales data, which is not always available, so an estimation error is not unlikely. Newman et al (2012) provide hotel data and describe the complexity of acquiring a proper data-set. Estimation errors lead to uncertainty in the parameters and very likely a misspecified model. Optimising a misspecified model leads to potentially suboptimal policies and revenue loss. A relatively recent field of optimisation that takes into account uncertainty in the optimisation procedure is robust optimisation. In this field, the values of the parameters are assumed to lie in an uncertainty set, rather than to be known exactly. Robust optimisation methods provide solutions where the worst-case scenario is optimised, providing a trade-off between risk and average reward. See Ben-Tal et al (2009) for an introduction of theories on robust optimization.

This paper studies a robust optimisation approach to single-leg choice-based revenue management. In this model, a single-leg revenue management problem is modelled as a Markov decision process and solved using dynamic programming. This paper provides a general robust formulation of this model. In each step of the dynamic program a small maximin problem has to be solved. The minimisation problem can be formulated as a linear program. The uncertain parameters are probabilities, of which the uncertainty set is modelled using a $\phi$-divergence measure. Tractable robust counterparts are presented for this problem. The methodologies that are used are based on Nilim and El Ghaoui (2005), who provide a robust formulation for general dynamic programming formulations, and the recent paper by Ben-Tal et al (2013), who provide a novel formulation for robust counterparts for probabilities. The main contribution of this paper is a tractable robust formulation for general choice models. Numerical results in section 'Numerical results' show that the robust solution method outperforms the nominal solution in many cases when using estimated parameters. Moreover, the robust solution method gives a relatively higher improvement in revenue for smaller hotels than for larger hotels. Related to this paper is Rusmevichientong and Topaloglu (2012), where a robust formulation is provided of the multinomial logit model. In contrast to their paper, this paper focuses on the estimated probabilities instead of the parameters of the chosen model. This way the robust solution method can be applied to any choice-model, or any estimate of the choiceprobabilities.

Other revenue management problems have been solved using robust optimisation. Several static and dynamic single-leg revenue management problems that do not take into account customer choice behaviour have been studied. Ball and Queyranne (2009) provide a robust solution method for the single-leg revenue management problem that does not require demand information. Robust results for various policy classes are provided. Birbil et al (2009) provide robust optimization methods for one static and one dynamic singleleg revenue management problem. Lan et al 
(2008) provide a robust solution method for the single-leg revenue management problem under independent demand model when limited information is available on demand. Network revenue management problems have also been studied in robust optimisation context. Among them, Lai and $\mathrm{Ng}$ (2005) provide a stochastic programming solution to a network model for hotels, without using choice-models. Perakis and Roels (2010) describe two robust solution methods for solving the network revenue management problem that does not take into account customer choice behaviour. One solution method solves the maximin problem and the other solves the minimax regret problem. As mentioned before, Rusmevichientong and Topaloglu (2012) provide a study of robust optimization applied to the assortment problem under the multinomial logit model. Farias et al (2013) provide a robust non-parametric estimation method and a study on selecting the right choice-model using sales data.

The remainder of this paper is organised as follows: In section 'Introduction to robust linear optimisation and $\phi$-divergence uncertainty sets', an introduction is given to robust linear optimisation. Moreover, the theory of $\phi$-divergence uncertainty sets is explained, which is used in the theories of this paper. In section 'Model description', the single-leg customer choice model is described. The extension to cancellations is also presented. The model is reformulated in an equivalent formulation that is more convenient for this paper. In section 'Robust reformulation', the robust counterpart of the nominal model is presented. First the formulation of the uncertainty sets is given. Then the robust dynamic program is formulated. The remainder of this section provides tractable reformulations for several $\phi$-divergence measures. In section 'Numerical results', numerical results are presented to validate the model. In section 'Concluding Remarks' some concluding remarks are given.

\section{MODEL DESCRIPTION}

In this section, the set-up of the problem is presented in hotel-context. However, the general description applies to other areas, such as seats on a flight or tickets for a theatre performance. Consider a hotel with $C$ identical rooms that wants to sell them in $T$ time units, 0 being the arrival time. This arrival time is typically an arrival day, where the rooms for that night are offered for sale $T$ days in advance. Overbooking is allowed up to $C_{\max }$ rooms. Each room can be sold using a fare product $j$, which is a combination of a room with a price $r_{j}$ and certain conditions, such as the cancellation policy. Assume there is a finite number of fare products $N=\{1, \ldots, n\}$. At each moment in time the hotel manager decides which offerset $S \subset N$ of fare products to offer. Potential customers arrive according to a Poisson process with rate $\lambda>0$. These clients show interest in the hotel, but their final decision is based on the offer-set $S$ displayed. The customer either buys one of the fare products $j \in S$, with probability $P_{j}(S)$, or leave and buy nothing at all, with probability $P_{0}(S)$. Customers are allowed to cancel their reservation, according to the cancellation policy. Assume that the cancellation of reservations happens independent from each other. This assumption is intuitively clear: clients are assumed to arrive independently, so their decision to cancel their reservation is not dependent on the decisions of other clients. If there are $x_{j}$ reservations for fare product $j$, then cancellations occur according to the exponential distribution with rate $\gamma_{j} x_{j}$, $\gamma_{j} \in \mathbb{R}^{+}$. See Figure 1 for an overview of the model.

The problem for the manager is to decide which offer-set $S \subset N$ to offer to maximise expected revenue. To solve this problem, the continuous time Markov decision process is discretised and set up as a dynamic programming formulation. The state space of this dynamic programming formulation keeps 


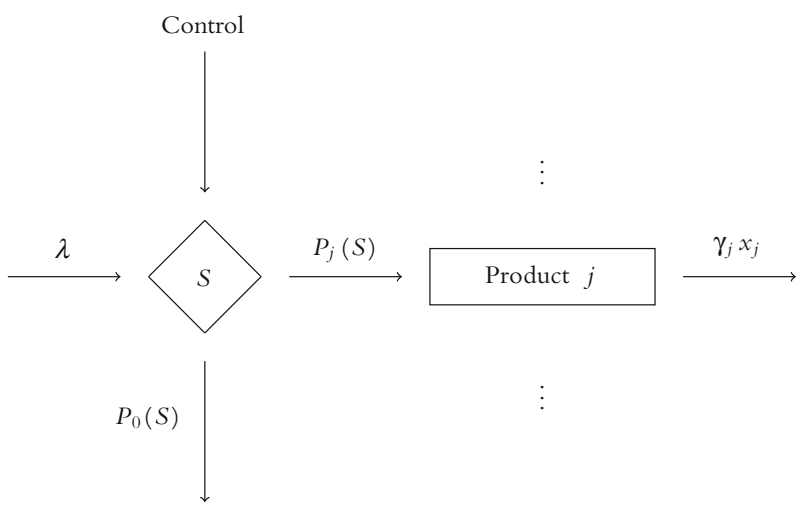

Figure 1: Visualisation of the customer choice cancellation model. Per arrival day the arrival process is Poisson distributed with parameter $\lambda$. The manager controls the offer set $S$. Under this offer set an arriving customer buys product $j \in S$ with probability $P_{j}(S)$. With probability $P_{0}(S)$ the customer buys nothing. Finally, cancellations of product $j$ follow an exponential distribution with parameter $\gamma_{j}$.

track of the number of reservations per product, so it grows exponentially with the number of products. Previous studies found a solution method that approximates the optimal solution by collapsing the state space (Algorithm 3.1 in Sierag et al 2015). This is the only available tractable solution method that shows promising results. Therefore, this algorithm, which is called nominal method henceforth, is used as a benchmark and functions as a base for the robust solution method that is to follow. Another approximation described in Sierag et al (2015) considers the model without cancellations. It is based on Talluri and van Ryzin (2004), and is denoted by Talluri and van Ryzin (TvR) method henceforth. The TvR method is also included in the numerical studies.

The collapsed dynamic program only keeps track of the total number of reservations, leading to the state space $\left\{0,1, \ldots, C_{\max }\right\}$. Time is divided into $T$ time periods, where the length of the intervals is such that the probability that more than one event occurs is very small. Therefore, it is assumed that only one event occurs per time period, where an event is either an arrival, a cancellation, or neither arrival nor cancellation. Denote with $\lambda$ the probability that a customer arrives in a time period, and $\gamma y$ the probability that a product is cancelled in state $\gamma, \gamma \in \mathbb{R}$. The single cancellation rate can be estimated from the individual cancellation rates, for example by the average, as is suggested in Sierag et al (2015). The probability that no purchase occurs in a time period equals the sum of the probability that neither an arrival and nor a cancellation occurs, and the probability that an arrival occurs but the arriving customer makes no purchase. This is equal to

$$
(1-\lambda-\gamma \gamma)+\lambda P_{0}(S)=1-\lambda \sum_{j \in S} P_{j}(S)-\gamma \gamma .
$$

In each time period, the decision needs to be made which set $S$ to offer. Note that time has to be scaled such that $\lambda+\gamma C_{\max } \leq 1$, otherwise the probabilities are not well defined.

Let $V_{t}(\gamma)$ be the maximal expected revenue from time $t$ to the arrival day in state $\gamma$. Define $\Delta H_{j}(t)$ by

$\Delta H_{j}(t)=\left\{\begin{array}{cc}\gamma_{j} c_{j}(t)+\left(1-\gamma_{j}\right) \Delta H_{j}(t-1) & \text { if } \mathrm{t}>1, \\ 0 & \text { if } \mathrm{t}=1,\end{array}\right.$

for all $j \in N$. The Bellman equation corresponding to the discretised Markov chain 


$$
V_{t}(\gamma)=\left\{\begin{array}{rlrl}
\max _{S \subset N} & \left\{\lambda \sum_{j \in S} P_{j}(S)\left[r_{j}-\Delta H_{j}(t)+V_{t-1}(\gamma+1)\right]\right. & & \\
& +\gamma \gamma V_{t-1}(\gamma-1) & & \\
& \left.+\left(1-\lambda \sum_{j \in S} P_{j}(S)-\gamma \gamma\right) V_{t-1}(\gamma)\right\} & & \\
& \gamma C_{\max } V_{t-1}\left(C_{\max }-1\right) & & \\
& +\left(1-\gamma C_{\max }\right) V_{t-1}\left(C_{\max }\right) & \text { if } \quad \mathrm{y}=\mathrm{C}_{\max }, \\
\max _{S \subset N} & \left\{\lambda \sum_{j \in S} P_{j}(S)\left[r_{j}-\Delta H_{j}(t)+V_{t-1}(1)\right]\right. \\
& \left.+\left(1-\lambda \sum_{j \in S} P_{j}(S)\right) V_{t-1}(0)\right\} & & \text { if } \quad \mathrm{y}=0 .
\end{array}\right.
$$

isBelow a reformulation of equation (1) is given, for convenience later on in the paper. Note that $\lambda, P_{j}(S)$ and $\gamma$ are probabilities. Define $\tilde{P}_{j}(S)$ : $=\lambda P_{j}(S)$ for all $j \in N\left(P_{j}(S)=0\right.$ if $\left.j \notin S\right)$, $\tilde{P}_{n+1}(S):=\gamma \gamma$, and $\tilde{P}_{0}(S)=1-\sum_{j=1}^{n+1} \tilde{P}_{j}(S)$. Also define $\tilde{r}_{j}:=r_{j}-\Delta H_{j}(t)$. Then equation (1) can be rewritten as

$$
\begin{array}{r}
V_{t}(\gamma)=\max _{S \subset N}\left\{\sum_{j \in S} \tilde{P}_{j}(S)\left[\tilde{r}_{j}+V_{t-1}(\gamma+1)\right]\right. \\
\left.+\tilde{P}_{n+1}(S) V_{t-1}(\gamma-1)+\tilde{P}_{0}(S) V_{t-1}(\gamma)\right\} .
\end{array}
$$

\section{ROBUST REFORMULATION}

Finding accurate estimates for the problem at hand is challenging. Commonly used maximum likelihood estimation methods can be found in Newman et al (2014), Sierag et al (2015), or Talluri and van Ryzin (2004). Estimating parameters from data often leads to estimation errors, as is the case with the mentioned methods. Incorrect estimates may lead to suboptimal decisions, and therefore suboptimal revenue. One way to deal with estimation errors in the optimisation process is robust optimisation. In this field, the values of the parameters are assumed to lie in an uncertainty set, rather than to be known exactly. The worst-case scenario is optimised under the uncertainty set. The goal is to improve performance by using the robust solution method rather than the nominal solution method. In (6) a brief introduction to robust linear optimisation is given.

Recently, Ben-Tal et al (2013) have provided tractable robust counterpart formulations for uncertainty sets that are based on $\phi$-divergence measures. These uncertainty sets are used to model uncertainty in probabilities. The dynamic program in equation (2) uses probabilities consisting of estimated parameters to find an optimal solution. The derivation of tractable robust counterparts for (2) under $\phi$ divergence uncertainty sets is described below.

\section{Reformulation}

The uncertainty in the adjusted purchase probabilities $\tilde{P}_{j}(S)$ is assumed to deviate from the nominal value $\bar{p}$ according to a $\phi$-divergence measure. Consider a function $\phi: \mathbb{R} \rightarrow \mathbb{R}$ that is convex for $t \geq 0, \quad \phi(1)=0, \quad 0 \phi(1 / 0):=$ $a \lim _{t \rightarrow \infty} \phi(t) / t$ for $a>0$, and $0 \phi(0 / 0):=0$. The $\phi$-divergence measure $I_{\phi}(p, q)$ between two vectors $p$ and $q, p, q \in \mathbb{R}^{n}$, is defined as 


$$
I_{\phi}(p, q):=\sum_{i=1}^{n} q_{i} \phi\left(p_{i} / q_{i}\right) .
$$

A $\phi$-divergence measure measures the distance between two (probability) vectors according to the divergence function $\phi$.

A popular choice for $\phi$ is the Cressie-Read divergence:

$$
\phi^{\theta}(t)=\frac{1-\theta+\theta t-t^{\theta}}{\theta(1-\theta)}, \quad \theta \neq 0,1, \quad t \geq 0 .
$$

The $\phi$-divergence measure $I_{\phi}(p, q)$ is then given by

$$
I_{\phi}(p, q)=\frac{1}{\theta(1-\theta)}\left(1-\sum_{i} p_{i}^{\theta} q_{i}^{1-\theta}\right) .
$$

In section 'Discussion of selected $\phi$-divergence measures', other $\phi$-divergence measures are discussed.

In Ben-Tal et al (2013), it is shown that the robust counterpart of a linear constraint with $\phi$-divergence uncertainty can be written in terms of the conjugate $\phi^{*}: \mathbb{R} \rightarrow \mathbb{R} \cup \infty$ of $\phi$, which is defined as follows:

$$
\phi^{*}(s):=\sup _{t \geq 0}\{s t-\phi(t)\} .
$$

Let $X$ be a one-dimensional random variable with finite support $\{1, \ldots, m\}$. Denote $p=$ $\left(p_{1}, \ldots, p_{m}\right)$ as the probability vector of $X$, such that $p_{i}=\mathbb{P}(X=i)$. Let $\hat{p}_{0}$ be the maximum likelihood estimator of $p$. From Ben-Tal et al (2013), the uncertainty region for $p$ is given by

$$
\left\{p \in \mathbb{R}^{m} \mid I_{\phi}\left(p, \hat{p}_{0}\right) \leq \rho\right\},
$$

with

$$
\rho:=\frac{\phi^{\prime \prime}(1)}{N} \chi_{m, 1-\alpha}^{2},
$$

with $N$ the sample size, $\alpha$ the confidence level, and $\chi_{m, 1-\alpha}^{2}$ is the $1-\alpha$ percentile of the $\chi_{m}^{2}$ distribution. Then the uncertainty set $Z_{S}$ for the purchase probabilities $\tilde{P}_{j}(S)$ is given by

$$
Z_{S}=\left\{p \in \mathbb{R}^{n+2} \mid p \geq 0, C p \leq d, I_{\phi}(p, \bar{p}) \leq \rho\right\},
$$

with

$$
\begin{aligned}
C_{\bullet j} & =\left\{\begin{array}{cc}
(1,-1) & \text { if } \mathrm{j} \in \mathrm{S} \cup\{0, \mathrm{n}+1\}, \\
(0,0) & \text { otherwise. }
\end{array}\right. \\
d & =(1,-1) .
\end{aligned}
$$

Nilim and El Ghaoui (2005) provide a robust formulation of dynamic programming with uncertainty in the probabilities. From their analysis, it follows that the nominal dynamic program (1) under uncertainty sets $Z_{S}$ can be solved using the following recursive formula:

$$
V_{t}(\gamma)=\max _{S \subset N} \Phi(S) .
$$

with

$$
\begin{aligned}
& \Phi(S)=\min \left\{\sum_{j \in S} p_{j}\left[\tilde{r}_{j}+V_{t-1}(\gamma+1)\right]\right. \\
& \left.+p_{n+1} V_{t-1}(\gamma-1)+p_{0} V_{t-1}(\gamma) \mid p \in Z_{S}\right\}
\end{aligned}
$$

The uncertainty problems $\Phi(S)$ can be solved for each $S$ independently. When $\Phi(S)$ is known for all $S$, then optimisation problem (4) becomes a maximisation problem over a finite set of integers. The challenge is to evaluate $\Phi(S)$. For this purpose, it is notationally convenient to move the formula in the objective of (5) to the constraints, by setting the objective to minimise to $t \in \mathbb{R}$ and add the constraint

$$
\begin{aligned}
& \sum_{j \in S} p_{j}\left[\tilde{r}_{j}+V_{t-1}(\gamma+1)\right]+p_{n+1} V_{t-1}(\gamma-1) \\
& \quad+p_{0} V_{t-1}(\gamma) \leq t
\end{aligned}
$$

This leads to the following equivalent optimisation problem:

$$
\begin{aligned}
& \min \left\{t \in \mathbb{R} \mid \sum_{j \in S} p_{j}\left[\tilde{r}_{j}+V_{t-1}(\gamma+1)\right]\right. \\
& +p_{n+1} V_{t-1}(\gamma-1)+p_{0} V_{t-1}(\gamma)-t \leq 0, \\
& \left.\forall p \in Z_{S} \cdot\right\} .
\end{aligned}
$$


Define $x \in \mathbb{R}^{2}, a \in \mathbb{R}^{2}$, and $B \in \mathbb{R}^{2 \times(n+2)}$ as follows:

$$
\begin{aligned}
& x=\left(t, x_{0}\right), \\
& a=(-1,0), \\
& B=\left(\begin{array}{cc}
0 & 0 \\
V_{t-1}(y) & \tilde{r}_{1}+V_{t-1}(y+1)
\end{array}\right. \\
& \text { the constraint can be rewritten to } \\
& \begin{cases}0 \quad \geq(a+B p)^{\top} x, & \forall p \in Z_{S}, \\
x_{0} & =1 .\end{cases}
\end{aligned}
$$

The following property from Ben-Tal et al (2013) can now be applied.

Property 1 Theorem 4.1 in Ben-Tal et al (2013) Consider the linear constraint

$$
(a+B p)^{\top} x \leq b, \quad p \in Z,
$$

where $x \in \mathbb{R}^{n}$ is the vector to be optimised, $a \in \mathbb{R}^{n}, B \in \mathbb{R}^{n \times m}$ and $b \in \mathbb{R}^{n}$ are given parameters, $p \in \mathbb{R}^{m}$ is the uncertain parameter, and

$$
Z=\left\{p \in \mathbb{R}^{m} \mid p \geq 0, C p \leq d, I_{\phi}(p, q) \leq \rho\right\},
$$

is the uncertainty region of $p$ with $q \in \mathbb{R}_{+}^{m}$, $\rho>0, d \in \mathbb{R}^{k}$, and $C \in \mathbb{R}^{k \times m}$. Then a vector $x \in \mathbb{R}^{n}$ satisfies (7) if and only if there exist $\eta \in \mathbb{R}^{k}$ and $\xi \in \mathbb{R}$ such that $(x, \eta, \xi)$ satisfies

$$
\left\{\begin{array}{l}
a^{\top} x+d^{\top} \eta+\rho \xi+\xi \sum_{i} q_{i} \phi^{*}\left(\frac{B_{i}^{\top} x-C_{i}^{\top} \eta}{\xi}\right) \leq b, \\
\eta \geq 0, \xi \geq 0
\end{array}\right.
$$

where $B_{i}$ and $C_{i}$ are the $i$-th columns of $B$ and $C$, respectively, and $\phi^{*}$ is the conjugate function of $\phi$.

$$
\left.\begin{array}{ccc}
\cdots & 0 & 0 \\
\cdots & \tilde{r}_{n}+V_{t-1}(y+1) & V_{t-1}(y-1)
\end{array}\right) .
$$

The robust counterpart of (6) is then given by

$$
\begin{aligned}
& a^{\top} x+d^{\top} \eta+\rho_{P} \xi+\xi \sum_{i=0}^{n} \bar{p}_{i} \phi^{*}\left(\frac{B_{i}^{\top} x-C_{i}^{\top} \eta}{\xi}\right) \leq 0, \\
& \eta \geq 0, \\
& \xi \geq 0 .
\end{aligned}
$$

The robust counterparts for the boundaries (last two equations of (1)) are also given by equation (8), where the parameters are given by

$$
\begin{aligned}
& x=\left(t, x_{0}\right), \\
& a=(-1,0), \\
& B=\left(\begin{array}{cc}
0 & 0 \\
V_{t-1}(y) & V_{t-1}(y-1)
\end{array}\right), \\
& p\left(p_{0}, p_{n+1}\right),
\end{aligned}
$$

and

$$
\begin{aligned}
x & =\left(t, x_{0}\right), \\
a & =(-1,0), \\
B & =\left(\begin{array}{cccc}
0 & 0 & \ldots & 0 \\
V_{t-1}(\gamma) & \tilde{r}_{1}+V_{t-1}(\gamma+1) & \ldots & \tilde{r}_{n}+V_{t-1}(\gamma+1)
\end{array}\right), \\
p & =\left(p_{0}, p_{1}, \ldots, p_{n}\right),
\end{aligned}
$$


respectively.

A tractable reformulation of the robust counterpart under the Cressie-Read divergence is given by (see appendix of Ben-Tal et al, 2013)

$$
\left\{\begin{array}{l}
a^{\top} x+\eta_{1}-\eta_{2}+\xi \rho_{P}+\frac{\xi}{\theta} \sum_{i} \bar{p}_{i}\left(\left(\frac{y_{i}}{\xi}\right)^{\frac{\theta}{\theta-1}}-1\right) \leq 0 \\
y_{i}=\xi-(1-\theta)\left(B_{i}^{\top} x-\eta_{1}+\eta_{2}\right), \\
\eta \geq 0, \xi \geq 0 .
\end{array}\right.
$$

This problem can solved using conic quadratic programming (CQP). Tractable robust counterparts for other popular $\phi$-divergence measures can be found in Ben-Tal et al (2013).

The parameters of the uncertainty sets need to be estimated from data. For some parameters, the estimation procedure is trivial or already described. The parameter $\bar{p}$ follows directly from the maximum likelihood estimates, for example from the estimation procedure described in Newman et al (2014), Sierag et al (2015), or Talluri and van Ryzin (2004). The parameter $\rho_{P}$ is defined by equation (3).

\section{Discussion of selected $\phi$-divergence measures}

Next, tractable reformulations of (8) are provided for popular choices of $\phi$. Each measure is appropriate in a different situation. A motivation is given when to select which measure.

- Kullback-Leibler The Kullback-Leibler measure is

$$
I_{\phi_{k l}}(p, \bar{p})=\sum p_{i} \log \left(p_{i} / \bar{p}_{i}\right)
$$

This asymmetric measure punishes upward deviation of $p_{i}$ from $\bar{p}_{i}$ and rewards downward deviation. This measure is appropriate when the parameters are more likely to be smaller than the estimated parameters.

- Burg entropy The Burg entropy measure is given by

$$
I_{\phi_{b}}(p, \bar{p})=\sum \bar{p}_{i} \log \left(p_{i} / \bar{p}_{i}\right) .
$$

Also for this asymmetric measure it holds that it punishes upward deviation of $p_{i}$ from $\bar{p}_{i}$ and rewards downward deviation. The amplification of the punishment or reward is constant and dependent on the estimation of the nominal vector $\bar{p}$. The unfortunate property of this measure is that any value of $p_{i}$ is feasible: $p_{i}$ can be approximately zero, since it has a negative contribution to $I_{\phi_{b}}(p, \bar{p})$, and $p_{i}$ can be greater than $\bar{p}_{i}$, up to 1 , as long as $p_{j}$ is small enough, for some $j \neq i$.

- J-divergence The J-divergence measure leads to a $\phi$-divergence of

$$
I_{\phi_{j}}(p, \bar{p})=\sum\left(p_{i}-\bar{p}_{i}\right) \log \left(p_{i} / \bar{p}_{i}\right) .
$$

This asymmetric measure punishes both upward and downward deviation of $p_{i}$ from $\bar{p}_{i}$. The measure is similar to Kullback-Leibler and Burg Entropy, yet the factor $p_{i}-\bar{p}_{i}$ ensures that all deviation is punished and none rewarded. Larger deviation is punished heavier than small deviations.

- $\chi^{2}$-distance The $\phi$-divergence of the $\chi^{2}$ distance measure is given by

$$
I_{\phi_{c}}(p, \bar{p})=\sum \frac{\left(p_{i}-\bar{p}_{i}\right)^{2}}{p_{i}} .
$$

This measure punishes larger deviations more than smaller deviations. However, upward deviation is punished less than downward deviation because of the $1 / p_{i}$ term. The punishment is relative to the quantity of the vector $p$.

- Modified $\chi^{2}$-distance The $\phi$-divergence of the modified $\chi^{2}$-distance is given by

$$
I_{\phi_{m c}}(p, \bar{p})=\sum \frac{\left(p_{i}-\bar{p}_{i}\right)^{2}}{\bar{p}_{i}} .
$$

This measure also punishes larger deviations more than smaller deviations. However, in this case, upward deviation is punished equally as downward deviation because of the $\bar{p}_{i}$. The punishment is relative to the quantity of the nominal value $\bar{p}_{i}$. 
- Hellinger distance The $\phi$-divergence of the Hellinger distance is given by

$$
I_{\phi_{h}}(p, \bar{p})=\sum\left(\sqrt{p_{i}}-\sqrt{\bar{p}_{i}}\right)^{2} .
$$

This is the $l_{2}$-norm applied to the vector $\left(\sqrt{p_{1}}, \ldots, \sqrt{p_{n}}\right)$, which is a unit vector under the $l_{2}$-norm. This measure is more sensitive to changes in smaller probabilities than larger probabilities.

- $\chi$-divergence of order $\theta>1$ The $\phi$-divergence of the $\chi$-distance of order $\theta>1$ is given by

$$
I_{\phi_{c a}}(p, \bar{p})=\sum \bar{p}_{i}\left|1-p_{i} / \bar{p}_{i}\right|^{\theta} .
$$

This measure punishes larger deviations more than small deviations. How severe the deviations are punished depends on the parameter $\theta$. Larger $\theta$ punish deviations more than smaller $\theta$.

- Variation distance The $\phi$-divergence of the variation distance is given by

$$
I_{\phi_{\nu}}(p, \bar{p})=\sum\left|p_{i}-\bar{p}_{i}\right| .
$$

This is the $l_{1}$ norm. Larger deviation is punished linearly more than smaller deviations.

- Cressie and read The $\phi$-divergence for the Cressie and Read measure with parameter $\theta$ is given by

$$
I_{\phi_{h}}(p, \bar{p})=\frac{1}{\theta(1-\theta)}\left(1-\sum p_{i}^{\theta} \bar{p}_{i}^{1-\theta}\right) .
$$

This measure gives larger punishment to larger deviations if $\theta$ is large. For small $\theta$ the effect of large deviations is less severe.

\section{NUMERICAL RESULTS}

In this section, numerical results are provided to validate the robust solution method for the single-leg revenue management model with cancellations and overbooking. The benchmark policy is given by the nominal solution method described in section 'Model description'.
The model parameters that are used are based on Simulation Example 2 in Talluri and van Ryzin (2004) and the example used in the numerical results section of Sierag et al (2015). These parameters are thought to be realistic and based on observations in practice. Let $n=$ 10 be the number of products sold with corresponding price vector

$r=(240,220,190,160,120,112,96,80,74,70)$.

Demand, cancellation rates, and purchase probabilities are independent from the time period $t$. To allow the study of the effect of high volume in demand and low volume in demand the load factor $l$ is introduced. In the studies, the values $l \in\{0.6,0.8,1,1.2,1.4\}$ are used. Demand $\lambda$ per time unit is then defined as

$$
\lambda(C, l, T)=\frac{C l}{T} .
$$

A low value of $l$ implies a low demand relative to the capacity $C$ and a high value of $l$ implies a high demand relative to the capacity $C$.

Purchase probabilities are modelled by the multinomial logit model (MNL). The only attribute that is considered is price, but it is assumed that there exist high price-sensitive and low price-sensitive customers which have different parameters $\beta_{H}=-0.005$ and $\beta_{L}=-0.0015$, respectively (as in Talluri and van Ryzin, 2004; Sierag, et al, 2015). The nopurchase parameter is set to $\alpha=0$ such that the MNL model is the same as in Talluri and van Ryzin (2004) and Sierag et al (2015). Overbooking is allowed up to 20 per cent of the total capacity $C$ (this is more for computational reasons: the policies found will almost surely not open any product categories for sale as the overbooking is close to 20 per cent). Cancellation rates are assumed linear and the parameters $\gamma \in \mathbb{R}^{n}$ depend on $l$ and $T$ in the following way:

$$
\begin{aligned}
\gamma= & (18 / 25,8 / 25,14 / 25,7 / 25,1 / 5,9 / 25, \\
& 4 / 25,2 / 25,1 / 125,1 / 25) \frac{l}{T} .
\end{aligned}
$$


To evaluate the performance of both robust and nominal policies for a particular model instance, 1000 data sets are simulated. From each dataset, the parameters of the model are estimated, which are used by both solution methods. Simulation is used to estimate the revenue corresponding to a policy. The estimation errors are between 0.1 and 0.4 per cent.

\section{Hotel size}

First the performances of the robust solution method for different hotel sizes are compared. Moreover, different $\phi$-divergences are applied. The booking horizon is $T=1000$ time units and the sample size is 100 arrival days. See Table 1 for the results.

The results show that the robust solution methods perform better compared to the nominal solution method for small hotels. For larger the hotels, the difference is smaller. This might be the case because either the nominal solution performs better for larger hotels, or the robust solution methods perform worse for larger hotels. These results suggest that smaller hotels would relatively benefit more from using a robust solution method than larger hotels. This is an important observation, since most of the hotels are small and medium enterprise hotels and have a relatively small number of rooms.

The results show no significant difference in performance between the robust solution methods. Using any robust solution method seems better than using none. For a fixed hotel size, the divergences do not show much difference. Any difference might even be a caused by the small estimation errors. Also, whenever a divergence shows better performance for one hotel size, another divergence performs better for another hotel size. For example, for $C=$ 10 the $\chi^{2}$-divergence performs best, but for $C=20$ the divergence performs worst.

Note that the TvR approximation does not perform well, and so it does in the examples that follow. Also, no significant different in performance of $\phi$-divergences was found in the next examples. For convenience only the results of the Cressie-Read divergence are compared against the nominal method, and the TvR method is left out.

Table 1: Performance of nominal and robust solution methods for various hotel sizes

\begin{tabular}{|c|c|c|c|c|c|c|c|c|c|c|}
\hline \multirow[t]{3}{*}{$\phi$-div } & \multicolumn{10}{|c|}{ Hotel size } \\
\hline & \multicolumn{2}{|c|}{10} & \multicolumn{2}{|c|}{20} & \multicolumn{2}{|c|}{50} & \multicolumn{2}{|c|}{100} & \multicolumn{2}{|c|}{200} \\
\hline & Rev & $\begin{array}{l}\text { (per } \\
\text { cent) }\end{array}$ & Rev & $\begin{array}{l}\text { (per } \\
\text { cent) }\end{array}$ & Rev & $\begin{array}{l}\text { (per } \\
\text { cent) }\end{array}$ & $\operatorname{Rev}$ & $\begin{array}{l}\text { (per } \\
\text { cent) }\end{array}$ & Rev & $\begin{array}{l}(\text { per } \\
\text { cent })\end{array}$ \\
\hline Nominal & 1765 & & 3549 & & 8908 & & 16,711 & & 29,776 & \\
\hline TvR & 1749 & -0.91 & 3470 & -2.23 & 8235 & -7.56 & 15,209 & -8.99 & 26,355 & -11.49 \\
\hline Kullback-Leibler & 1791 & 1.47 & 3620 & 2.00 & 8948 & 0.45 & 16,793 & 0.49 & 29,781 & 0.02 \\
\hline Burg entropy & 1791 & 1.47 & 3621 & 2.03 & 8945 & 0.42 & 16,794 & 0.50 & 29,779 & 0.01 \\
\hline J-divergence & 1791 & 1.47 & 3621 & 2.03 & 8945 & 0.42 & 16,792 & 0.48 & 29,778 & 0.01 \\
\hline$\chi^{2}$-distance & 1792 & 1.53 & 3619 & 1.97 & 8946 & 0.43 & 16,793 & 0.49 & 29,784 & 0.03 \\
\hline $\begin{array}{l}\text { Modified } \chi^{2}- \\
\text { distance }\end{array}$ & 1791 & 1.47 & 3621 & 2.03 & 8946 & 0.43 & 16,796 & 0.51 & 29,780 & 0.01 \\
\hline Hellinger distance & 1791 & 1.47 & 3620 & 2.00 & 8945 & 0.42 & 16,794 & 0.50 & 29,777 & 0.00 \\
\hline $\begin{array}{l}\chi \text {-div. of order } \theta= \\
0.5\end{array}$ & 1792 & 1.53 & 3621 & 2.03 & 8946 & 0.43 & 16,793 & 0.49 & 29,781 & 0.02 \\
\hline Variation distance & 1791 & 1.47 & 3619 & 1.97 & 8947 & 0.44 & 16,795 & 0.50 & 29,783 & 0.02 \\
\hline Cressie-read & 1791 & 1.47 & 3620 & 2.00 & 8946 & 0.43 & 16,790 & 0.47 & 29,782 & 0.02 \\
\hline
\end{tabular}


Table 2: Performance of nominal and robust solution methods for various load factors

\begin{tabular}{lllllrrr}
\hline Load factor & \multicolumn{3}{c}{$C=20$} & & \multicolumn{2}{c}{$C=100$} \\
\cline { 2 - 3 } & Nom & Rob & (per cent) & & Nom & Rob & (per cent) \\
\hline 0.6 & 1934 & 1936 & 0.09 & 9107 & 9106 & -0.02 \\
0.8 & 2539 & 2553 & 0.56 & & 11,849 & 11,853 & 0.03 \\
1.0 & 3082 & 3125 & 1.39 & & 14,388 & 14,443 & 0.38 \\
1.2 & 3546 & 3619 & 2.03 & & 16,705 & 16,781 & 0.46 \\
1.4 & 3966 & 4039 & 1.82 & & 18,911 & 18,982 & 0.37 \\
\hline
\end{tabular}

Table 3: Performance of nominal and robust solution methods for a hotels of size $C=20$ and $C=100$ and different sample sizes

\begin{tabular}{lccccccc}
\hline Sample size & \multicolumn{3}{c}{$C=20$} & & \multicolumn{2}{c}{$C=100$} \\
\cline { 2 - 3 } & Nom & Rob & (per cent) & & Nom & Rob & (per cent) \\
\hline 1 & 3434 & 3473 & 1.12 & 15,510 & 15,536 & 0.17 \\
2 & 3286 & 3323 & 1.12 & & 15,472 & 15,512 & 0.26 \\
5 & 3482 & 3541 & 1.69 & & 16,442 & 16,496 & 0.33 \\
10 & 3513 & 3576 & 1.77 & & 16,646 & 16,705 & 0.35 \\
20 & 3542 & 3612 & 1.98 & & 16,665 & 16,729 & 0.38 \\
50 & 3545 & 3616 & 2.01 & & 16,690 & 16,760 & 0.42 \\
100 & 3546 & 3618 & 2.03 & & 16,708 & 16,777 & 0.41 \\
\hline
\end{tabular}

\section{Load factor}

The load factor influences the performance of the estimation method (see Sierag et al, 2015). Therefore, the robust solution method might perform different under different load factors. The parameters are set to $C=20$ and $C=100$ rooms, $T=100$ time periods, and the load factors that are used are $\{0.6,0.8,1,1.2,1.4\}$. The results are presented in Table 2.

The results show that under a small load factor the performance of the nominal solution and the robust solution are similar, while for larger load factors the robust solution outperforms the nominal solution. In practice, this means that the robust solution is preferred in popular areas or in high season, since it leads to significantly higher profits, while outside high season or at less popular locations the robust solution does not lead to higher profits.

\section{Sample size}

Now the behaviour of the solution methods according to different sample sizes is considered. One sample consists of the data collected for one arrival day. The uncertainty set parameter $\rho$ is evaluated accordingly. The size of the hotel is $C=20$ rooms and the booking horizon is $T=100$ time periods.

In Table 3 , the performance of the nominal and robust solution methods is provided. The results show that the average performance of the robust solution method outperforms the nominal solution method by 1-2 per cent for $C=20$, increasing as the sample size increases. For $C=100$ the performance is lower, up to 0.42 per cent.

\section{Unknown cancellation behaviour}

The robust solution method can be beneficial when the cancellation behaviour is not 
Table 4: Performance of nominal and robust solution methods for various hotel sizes

\begin{tabular}{lccccccc}
\hline Sample size & \multicolumn{3}{c}{$C=20$} & & \multicolumn{2}{c}{$C=100$} \\
\cline { 2 - 3 } & Nom & Rob & (per cent) & & Nom & Rob & (per cent) \\
\hline 1 & 3458 & 3544 & 2.48 & & 15,176 & 15,651 & 3.13 \\
2 & 3408 & 3484 & 2.22 & & 15,048 & 15,500 & 3.00 \\
5 & 3440 & 3524 & 2.44 & & 15,176 & 15,660 & 3.19 \\
10 & 3430 & 3515 & 2.47 & & 15,148 & 15,619 & 3.10 \\
20 & 3464 & 3559 & 2.74 & & 15,185 & 15,678 & 3.25 \\
50 & 3466 & 3559 & 2.70 & & 15,198 & 15,699 & 3.30 \\
100 & 3468 & 3561 & 2.68 & & 15,205 & 15,709 & 3.31 \\
\hline
\end{tabular}

Table 5: Performance of nominal and robust solution methods for different numbers of products

\begin{tabular}{|c|c|c|c|c|c|c|}
\hline \multirow[t]{2}{*}{ Number of products } & \multicolumn{3}{|c|}{$C=20$} & \multicolumn{3}{|c|}{$C=100$} \\
\hline & Nom & Rob & (per cent) & Nom & Rob & (per cent) \\
\hline 2 & 3472 & 3529 & 1.65 & 15,943 & 15,942 & -0.01 \\
\hline 3 & 3558 & 3628 & 1.97 & 16,615 & 16,684 & 0.41 \\
\hline 4 & 3549 & 3620 & 2.01 & 16,708 & 16,776 & 0.40 \\
\hline 5 & 3547 & 3621 & 2.08 & 16,706 & 16,778 & 0.43 \\
\hline 6 & 3548 & 3619 & 1.99 & 16,709 & 16,779 & 0.42 \\
\hline 7 & 3548 & 3620 & 2.03 & 16,707 & 16,777 & 0.42 \\
\hline 8 & 3550 & 3622 & 2.04 & 16,709 & 16,778 & 0.41 \\
\hline 9 & 3546 & 3621 & 2.12 & 16,709 & 16,781 & 0.43 \\
\hline 10 & 3546 & 3619 & 2.03 & 16,707 & 16,774 & 0.40 \\
\hline
\end{tabular}

known. When the cancellation parameter is equal to zero, the robust solution method can be used using this input parameter. The robust solution method then takes into account an uncertainty around the zero vector, which may lead to an improved result. This has been applied as follows: the hotel size is $C=20$ or $C=100$ rooms, the booking horizon is $T=$ 100 time periods, and the cancellation rate parameter is set to 0 . The results are given in Table 4.

The results show that the average performance is increased by about 2.5 per cent for $C=20$, while for $C=100$ the performance even increased by about 3.25 per cent. This strongly suggests that the robust solution method can successfully be applied when no information about cancellation behaviour is known. This is an important observation since knowledge on cancellations is not always available in practice.

\section{Number of products}

The performance of the solution methods may be influenced by the number of products $n$. The next example shows the performance under a different number of products. The hotel size is $C=20$ or $C=100$ rooms, the load factor is $l=1.2$, and the booking horizon is $T=100$ time periods. The results are presented in Table 5 .

From the results, it does not seem that the number of products has a big impact on the performance of the robust solution method. The robust solution method performs on average 1.65-2.12 per cent better for $C=20$, and 0.4 per cent for $C=100$. The results 
show very robust revenues against different numbers of products, which is preferred.

\section{CONCLUDING REMARKS}

Estimating parameters of a revenue management model that takes into account customer choice behaviour likely leads to estimation errors. One method to improve performance is using robust optimisation to take into account the estimation error. In this paper, a robust solution method is described for the customer choice cancellation model by Sierag et al (2015).

The dynamic programming formulation is converted into a robust dynamic program. The uncertain parameters, which are probabilities, are modelled using $\phi$-divergence uncertainty sets. In the dynamic program, small minimisation problems containing the uncertain parameters have to be solved. Novel robust optimisation methodologies from Ben-Tal et al (2013) lead to tractable solutions for several $\phi$ divergence measures.

In the numerical studies, it is shown that the robust solution method surprisingly performs better for smaller hotels than for larger hotels. As a consequence, this solution method is preferred for small and medium enterprise hotels, to which most hotels belong. Also, promising results are shown when cancellation behaviour is not known. The robust solution method outperforms the nominal solution method by up to 2 per cent when using estimated parameters. The performance also shows to be robust under different numbers of products. The facts that the robust solution method is tractable and provides good results make it attractive to be applied in practice.

The results in this paper can serve as a foundation for several topics further research. First, the methodology of this paper can be extended to network revenue management models that use choice models. The effects of parameter estimation errors in these network models can be studied and solved using robust optimisation. Solving network problems is not straight forward, so an intense study is necessary. Second, an extensive study of the performance of different $\phi$-divergence measures under different circumstances can provide promising insights. Each $\phi$-divergence measure includes and excludes different distances from the estimated parameters, which may give insight to which measure is preferred in which situation.

\section{REFERENCES}

Ball, M., and Queyranne, M. (2009). Toward robust revenue management: Competitive analysis of online booking. Operations Research, 57(4), 950-963.

Ben-Tal, A., den Hertog, D., De Waegenaere, A., Melenberg, B. and Rennen, G. (2013). Robust solutions of optimization problems affected by uncertain probabilities. Management Science, 59(1), 341-357.

Ben-Tal, A., El Ghaoui, L., and Nemirovski, A. (2009). Robust optimization. Princeton University Press.

Birbil, S., Frenk, J., Gromicho, J., and Zhang, S. (2009). The role of robust optimization in single-leg airline revenue management. Management Science, 55(1), 148-163.

Farias, V., Jagabathula, S., and Shah, D. (2013). A nonparametric approach to modeling choice with limited data. Management Science, 59(2), 305-322.

Lai, K., and Ng, W. (2005). A stochastic approach to hotel revenue optimization. Computers $\mathcal{E}$ Operations Research, 32(5), 1059-1072.

Lan, Y., Gao, H., Ball, M., and Karaesmen, I. (2008). Revenue management with limited demand information. Management Science, 54(9), 1594-1609.

Liu, Q., and van Ryzin, G. (2008). On the choice-based linear programming model for network revenue management. Manufacturing \& Service Operations Management, 10(2), 288-310.

Newman, J., Ferguson, M., and Garrow, L. (2012). Estimating discrete choice models with incomplete data. Transportation Research Record 2302, 130-137.

Newman, J. P., Ferguson, M. E., Garrow, L. A., and Jacobs, T. L. (2014). Estimation of choice-based models using sales data from a single firm. Manufacturing \& Service Operations Management, 16(2), 184-197.

Nilim, A., and El Ghaoui, L. (2005). Robust control of markov decision processes with uncertain transition matrices. Operations Research, 53(3), 780-798.

Perakis, G., and Roels, G. (2010). Robust controls for network revenue management. Manufacturing \& Service Operations Management, 12(1), 56-76.

Rusmevichientong, P., and Topaloglu, H. (2012). Robust assortment optimization in revenue management under the multinomial logit choice model. Operations research, 60(4), 865-882.

Sierag, D., Koole, G., van der Mei, R., van der Rest, J., and Zwart, B. (2015). Revenue management under customer 
choice behaviour with cancellations and overbooking. European Journal of Operational Research, 246(1), 170-185.

Strauss, A., and Talluri, K. (2012). A tractable consideration set structure for network revenue management. Working Paper.

Talluri, K., and van Ryzin, G. (2004). Revenue management under a general choice model of consumer behavior. Management Science, 50(1), 15-33.

\section{APPENDIX: INTRODUCTION TO ROBUST LINEAR OPTIMISATION AND $\phi$ - DIVERGENCE UNCERTAINTY \\ SETS}

Consider a problem that can be modelled as a linear program:

$$
\min \left\{c^{\top} x \mid A x \leq b\right\},
$$

with $c \in \mathbb{R}^{n}$ the cost vector, $x \in \mathbb{R}^{n}$ the vector of decision variables, $A \in \mathbb{R}^{m \times n}$ and $b \in \mathbb{R}^{m}$. The robust counterpart is given by

$$
\min \left\{c^{\top} x \mid A x \leq b, A \in Z\right\},
$$

with $c \in \mathbb{R}^{n}$ the cost vector, $x \in \mathbb{R}^{n}$ the vector of decision variables, $A \in \mathbb{R}^{m \times n}$ the uncertain parameters in uncertainty set $Z \subset \mathbb{R}^{m \times n}$ and $b \in \mathbb{R}^{m}$ a constant vector. Ben-Tal et al (2009) show that without loss of generality it may be assumed that only uncertainty in $A$ exists, and not in the objective $c$ or the right-hand-side $b$. Furthermore, Ben-Tal et al (2009) show that the uncertainty can be approached constraintwise, which is of the form

$$
(a+B \zeta)^{\top} x \leq b, \quad \forall \zeta \in Z,
$$

with the nominal value $a \in \mathbb{R}^{n}$ constant, $B \in$ $\mathbb{R}^{n \times m}$ constant, $\quad b \in \mathbb{R}$ constant, $\quad \zeta \in \mathbb{R}^{m}$ uncertain, and $Z$ the uncertainty region for $\zeta$.

Tractable formulations of the robust counterpart for several standard uncertainty regions are provided in Ben-Tal et al (2009). 